\title{
Correction to: The use of clays for chlorhexidine controlled release as a new perspective for longer durability of dentin adhesion
}

Lívia Rodrigues de Menezes ${ }^{1}$ - Emerson Oliveira da Silva ${ }^{1}$ - Lizandra Viana Maurat da Rocha ${ }^{1}$. Isabel Ferreira Barbosa ${ }^{2}$ - Marina Rodrigues Tavares ${ }^{1}$

Published online: 5 March 2020

(c) Springer Science+Business Media, LLC, part of Springer Nature 2020

\section{Correction to: Journal of Materials Science:} Materials in Medicine

https://doi.org/10.1007/s10856-019-6344-5

The original version of this article unfortunately contained a mistake. The affiliation of the author Marina Rodrigues
Tavares should read as "Instituto de Macromoléculas Professora Eloisa Mano da Universidade Federal do Rio de Janeiro UFRJ, Rio de Janeiro, Brazil".

The original article can be found online at https://doi.org/10.1007/ s10856-019-6344-5

Lívia Rodrigues de Menezes

liviarmenezes@ima.ufrj.br

1 Instituto de Macromoléculas Professora Eloisa Mano da Universidade Federal do Rio de Janeiro UFRJ, Rio de Janeiro, Brazil

2 Faculdade de Odontologia de Piracicaba/Universidade Estadual de Campinas UNICAMP, Campinas, Brazil 